\title{
Passive Observations of a Large DNS Service: 2.5 Years in the Life of Google
}

\author{
Wouter B. de Vries \\ University of Twente \\ w.b.devries@utwente.nl
}

\author{
Roland van Rijswijk-Deij \\ University of Twente and SURFnet bv \\ r.m.vanrijswijk@utwente.nl
}

\author{
Pieter-Tjerk de Boer \\ University of Twente \\ p.t.deboer@utwente.nl
}

\author{
Aiko Pras \\ University of Twente \\ a.pras@utwente.nl
}

\begin{abstract}
In 2009 Google launched its Public DNS service, with its characteristic IP address 8.8.8.8. Since then, this service has grown to be the largest and most well-known DNS service in existence. The popularity of public DNS services has been disruptive for Content Delivery Networks (CDNs). CDNs rely on IP information to geo-locate clients. This no longer works in the presence of public resolvers, which led to the introduction of the EDNS0 Client Subnet extension. ECS allows resolvers to reveal part of a client's IP address to authoritative name servers and helps CDNs pinpoint client origin. A useful side effect of ECS is that it can be used to study the workings of public DNS resolvers.

In this paper, we leverage this side effect of ECS to study Google Public DNS. From a dataset of 3.7 billion DNS queries spanning 2.5 years, we extract ECS information and perform a longitudinal analysis of which clients are served from which Point-of-Presence. Our study focuses on two aspects of GPDNS.

First, we show that while GPDNS has PoPs in many countries, traffic is frequently routed out of country, even if that was not necessary. Often this reduces performance, and perhaps more importantly, exposes DNS requests to state-level surveillance.

Second, we study how GPDNS is used by clients. We show that end-users switch to GPDNS en masse when their ISP's DNS service is unresponsive, and do not switch back. We also find that many e-mail providers configure GPDNS as the resolver for their servers. This raises serious privacy concerns, as DNS queries from mail servers reveal information about hosts they exchange mail with. Because of GPDNS's use of ECS, this sensitive information is not only revealed to Google, but also to any operator of an authoritative name server that receives ECS-enabled queries from GPDNS during the lookup process.
\end{abstract}

\section{INTRODUCTION}

The Domain Name System (DNS) is an important part of what the Internet is today. It resolves domain names to IP addresses. The DNS is a hierarchical system where different name servers are responsible for different parts of a domain name. In order to resolve a domain name, a socalled recursive resolver queries each name server responsible for part of a domain in turn, until it has the final answer. A customer of an Internet Service Provider (ISP), typically uses a recursive resolver that is provided by the ISP, and that is usually automatically configured. While most ISPs provide their own recursive resolver for their customers, it is usually not mandatory to use these. Customers can either run their own resolver, or use a third party resolver. Examples of organizations offering such third-party services include OpenDNS, Quad9 and Google. There are many reasons why an end-user might use a different resolver than the one operated by their ISP, such as stability, performance, privacy or to circumvent censorship.
In this paper we focus on one particular public resolver, Google Public DNS (GPDNS), which was launched in 2009. Since its inception, GPDNS has grown to be the largest public resolver in existence, serving hundreds of billions of requests per day [1]. While GPDNS uses only a few public IP addresses, its servers have a global presence. Google uses a technique called anycast to ensure traffic to GPDNS is routed to a nearby Point-of-Presence (PoP). This reduces latency for clients.

Now while services like GPDNS have a global presence, they typically do not have PoPs in every country. In fact, GPDNS currently has 21 active locations on 5 continents. It turns out that this poses challenges for Content Delivery Networks. CDNs frequently rely on the geo-location of the IP address of recursive resolvers as a proxy for the location of end customers. This information is then used to route requests to content caches near the end customer and to serve local content. The underlying assumption is that DNS queries are typically handled by a resolver 'near' the end customer, e. g., their ISP's resolver. If, however, a public resolver, such as GPDNS is used, this assumption breaks down, as requests appear to come from the PoP that handled a user request.

To address this problem an extension to the DNS called EDNSO Client Subnet (ECS) [2] was introduced. This extension allows recursive resolvers to include part of the IP address of the client that sent a query in requests to authoritative name servers. This can assist CDNs in more accurately determining where clients using public resolvers come from.

Interestingly, the use of ECS by name servers has unintended side effects. By sending ECS-enabled queries to CDNs that support the extension, it becomes possible to study the geographic distribution of their services, and how clients are mapped to certain services, as a number of existing studies have shown [3]-[7]. ECS, however, can also be used to examine how a public resolver works and is used. In this paper, we are the first to study a large scale public DNS resolver (GPDNS) over a 2.5-year period using passive observations of ECS data in DNS queries collected at a major authoritative name server.

The main contributions of our work are as follows:

- We show that while anycast routing is generally considered relatively stable [8], performance of Google's anycast network varies over time. Importantly, we show that traffic is frequently routed to out-of-country Points-of-Presence (PoPs), even if a local, in-country PoP is available. This potentially exposes DNS traffic to state-level surveillance. 


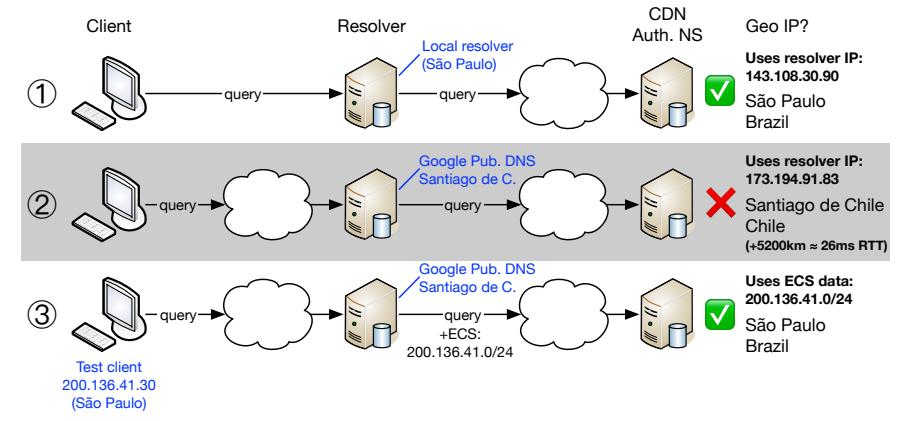

Figure 1. Explanation of EDNS0 Client Subnet

- We show that end-users switch away from their ISP's resolver if it is underperforming, and more importantly, that these users will not switch back.

- We show that there are SMTP servers that are configured to perform lookups through GPDNS. This is a potential privacy leak, in the sense that it allows the public resolver and any of the authoritative name servers involved to infer that there is likely communication between two parties.

- We make our full dataset covering 2.5 years and 3.7 billion queries available as open data to the research community at https://traces.simpleweb.org.

The rest of this paper is organized as follows: Section II provides background information. Then, Section III describes our methodology, including how data was collected. Next, Section IV contains data analysis and results. Section V discusses related work. Finally, Section VI provides conclusions and an outlook on future work.

\section{BACKGROUND}

\section{A. EDNS and EDNS Client Subnet}

The original DNS protocol [9], [10] puts limits on both the size of DNS responses (512 bytes in a UDP datagram) and what options and flags a DNS message can have. Many modern applications of the DNS have requirements that exceed the limits of the original protocol. For this reason, the Extension Mechanisms for DNS (EDNS0) [11] were introduced. EDNS0 uses a special DNS pseudo-record in the additional section of a DNS query or response. This so-called OPT record specifies EDNS parameters (e.g., the maximum message size), can be used to specify additional flags (e.g., DNSSEC flags) and can be used to specify new DNS options. The latter, the options, are encoded in the form of <tag,value $>$ pairs and can, for example, be used to convey metadata about a DNS message.

Many CDNs and other applications make use of the IP address from which queries are made to their authoritative DNS servers. This IP address is used as a proxy for the location of an end customer. It is used to perform a so-called Geo IP lookup, to determine (roughly) where a customer is coming from, and is used to make decisions about, e.g., which content to serve or whether or not to allow access to certain content. With the advent of public DNS resolvers, such as Google Public DNS, this model of identifying where clients are coming from runs into problems. The reason for this is that authoritative name servers for the CDN will now see queries as coming from GPDNS. Even if Geo IP databases are accurate enough to identify the country in which the GPDNS servers that handled the request are located, this may not provide the information to pinpoint the origin region of a request with sufficient accuracy.

To remedy this, the EDNS Client Subnet (ECS) option [2] was introduced. This option can be used by DNS resolvers to provide information about where a query originated. To do this, a DNS resolver includes two fields in the ECS option: the IP prefix from which the query originated and a source prefix length field that specifies the size of the provided prefix (e.g. /24). For privacy reasons, DNS resolvers typically limit how specific the scope is that they send in a request. The ECS standard [2] recommends using a maximum scope of / 24 for IPv4 and / 56 for IPv6. An authoritative name server can then use this information to decide which region-specific response to return to a query. To ensure that responses from the authoritative name servers are only cached for users in the correct prefix, the authoritative name server also includes its own scope prefix length field in the response. This field must be used by the DNS resolver when caching the response. For more information on what DNS servers should do in case of prefix-length mismatches, we refer to the RFC [2].

Figure 1 shows an example of 1) a local resolver, 2) a public resolver without ECS and 3) a public resolver with ECS. The figure shows the potential impact of not using ECS for a public resolver. The example is based on a client we control, located in São Paulo, Brazil. Without ECS, a CDN using Geo IP will assume this client is in Santiago de Chile, $2600 \mathrm{~km}$ away as the crow flies, adding a potential $26 \mathrm{~ms}$ to each network round-trip.

\section{Methodology}

As discussed in the introduction, EDNS Client Subnet provides a unique opportunity to observe the behaviour of large public DNS resolvers. In this section, we outline how we collected our data, and how we will use this to study one particular public DNS resolver operator: Google.

\section{A. Data collection}

1) Collection point: We used one of the authoritative name servers of SURFnet, the National Research and Education Network (NREN) in The Netherlands, to passively collect DNS queries from Google Public DNS. Collection started at the end of June 2015, and continues to the present day. Only DNS queries that include an ECS option are collected, and for these queries, we record the origin IP of the query (i.e. the IP of the Google resolver that sent the query), and the IP prefix and source prefix length included in the ECS option. In addition to this, we use CAIDA's IP prefix to Autonomous System (AS) dataset [12] to map the ECS IP prefix to an AS and we use the free IP2Location dataset to map the ECS IP prefix to a country.

The SURFnet name server we used is authoritative for approximately 10,000 DNS zones, including a number of 
Table I

Distribution of Google PoPs (State of 8 November 2017) AND RECEIVED QUERIES AT OUR AUTHORITATIVE NAME SERVER

\begin{tabular}{lrrrr}
\hline Continent & PoPs & Prefixes IPv4 & IPv6 & \# of queries \\
\hline Asia & 4 & 13 & 4 & $391,523,557$ \\
Europe & 6 & 19 & 6 & $1,800,743,147$ \\
North America & 8 & 40 & 8 & $1,450,006,164$ \\
Oceania & 1 & 3 & 1 & $2,633,248$ \\
South America & 2 & 3 & 2 & $29,143,338$ \\
\hline Total & 21 & 78 & 21 & $3,711,406,022$ \\
\hline
\end{tabular}

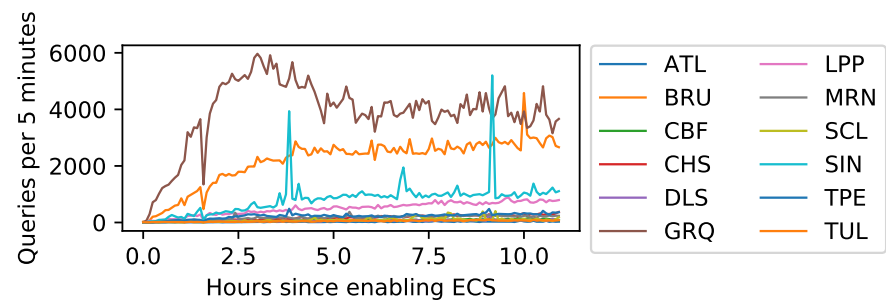

Figure 2. Ramp-up of GPDNS detecting and enabling ECS

popular public suffices ${ }^{1}$ such as .ac.uk, .gov.uk and . ac.be. As a result of this, this name server sees a wide spread of queries from all over the Internet and world. Note though, as we discuss in more detail below in Section III-C, we do expect bias in which Google PoPs send traffic to this server, due to resolver-to-authoritative RTT optimisation. Table I shows an overview of the data we collected for this study, broken down per continent from which queries originated.

2) BIND patch: Google Public DNS automatically detects support for ECS on authoritative name servers. In order to do this, Google regularly sends probing queries that include an ECS option. If an authoritative name server includes an ECS option in the response, this is interpreted as an indication of support. To ensure that Google would detect our name server as ECS-capable, we implemented a patch for the popular BIND DNS implementation. After this patch, BIND will accept the ECS option, and will include an ECS option in the response that mirrors the source address and prefix length in the scope prefix length field. Figure 2 shows the number of ECS-enabled queries per 5 minutes from Google PoPs increasing after we have enabled ECS on our patched name server as resolvers at these PoPs detect support for ECS.

3) Ethical Considerations: As the ECS standard [2] already specifies, there are inherent privacy concerns in the protocol, as a resolver that supports ECS includes a (sometimes significant) part of the client's IP address in queries. We are interested in how clients are routed to GPDNS and in general terms how GPDNS is used at the network operator level. Therefore, to protect user privacy, we take two measures: 1) we do not store query names, we only record query types and 2) we aggregate ECS client IP prefixes at the AS level when analysing the data, with one exception; if we believe the prefix contains

\footnotetext{
${ }^{1}$ For an explanation of public suffices, see https://publicsuffix.org.
}

servers that use GPDNS (rather than individuals), we analyse if certain types of hosts (specifically, e-mail servers) exist in these prefixes (Section IV-D).

A secondary concern is the effect on query and cache efficiency. While we implement ECS on the authoritative name server where we collect data, we do not differentiate DNS responses based on ECS. Since DNS resolvers that implement ECS should cache responses based on the ECS information, this may impact caching efficiency. Consequently, GPDNS may have to cache responses from our patched name server for every client prefix they send in ECS-enabled queries. The standard [2], however, provides clear guidelines for resolver implementers to avoid cache pollution. In addition to this, the impact of us implementing ECS will only have a limited impact, as the other authoritative name servers for domains for which our patched name server is authoritative do not implement ECS. In many cases this means only one in four queries sent from Google will result in an ECS-enabled response (of course depending on how Google's resolvers distribute queries over the set of authoritative name servers for a domain).

\section{B. Resolver IP to Point-of-Presence mapping}

While end-users query Google Public DNS via the frontend IP addresses 8.8.8.8 and 8.8.4.4 or their IPv6 counterparts, an individual Google DNS resolver then uses different IP addresses to actually resolve the query. The IP-prefixes that are used are published and are frequently updated [13]. Google identifies their PoPs using the three-letter IATA code of the nearest airport. During the first part of the measurement period we did not store the mapping of prefixes to Google PoPs, but we recovered it using The Wayback Machine $(\mathrm{TWM})^{2}$. Specifically, we collected 25 mappings between the start of our measurement period and the 3rd of August 2017 through TWM. From that point onward, we collected the mapping on a daily basis directly from Google through a DNS query, as described in Google's FAQ [13].

Figure 3 shows the number of prefixes associated with each PoP and how this varies over time. As the figure shows, several new PoPs were added over the period covered by our dataset, for example, approximately halfway through 2017 the Sydney PoP was added. We also observe, over the total duration, 4 instances where a prefix was reassigned to a different PoP. It is likely that due to the significant delay in mappings which we obtained via TWM we mismapped a portion of the traffic when such a reassignment occured. However, considering the large amount of total prefixes, the vast majority of which were not reassigned at any point, the impact of this is likely to be small. For our study, we use the prefix-to-PoP mapping we recorded to map queries to Google PoPs based on the prefix in which the source IP of a query is contained. In total we were unable to map 26,548,020 queries to their corresponding PoP $(0.7 \%$ of all queries in our dataset $)$.

\footnotetext{
${ }^{2}$ https://archive.org/
} 


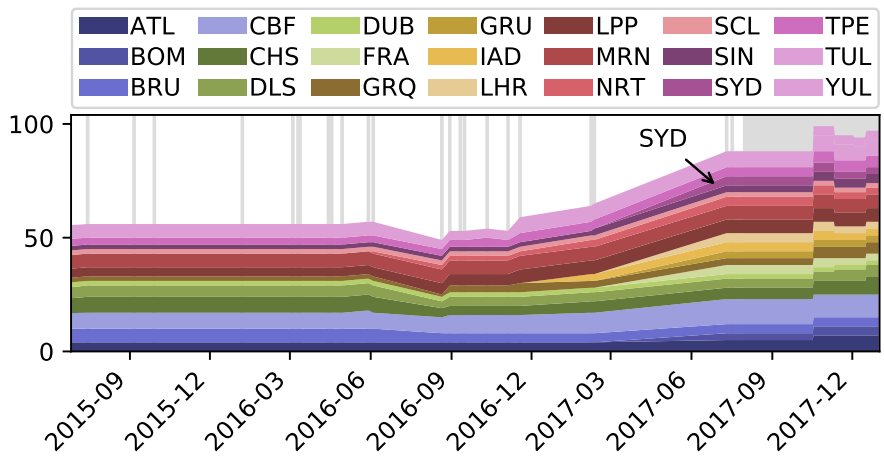

Figure 3. Number of prefixes associated with each of Google Public DNS PoPs. The gray background indicates where we have data.

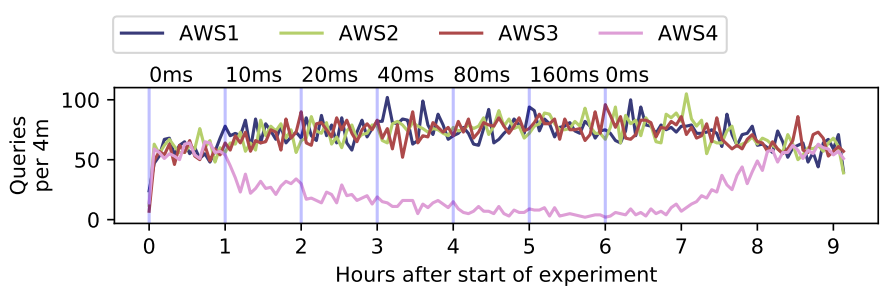

Figure 4. Division of queries from Google over 4 name servers. Every vertical line indicates a change in latency of AWS4 (e.g. $+10 \mathrm{~ms},+20 \mathrm{~ms}$ ).

\section{Distribution of queries over authoritative name servers}

As we discussed in Section III-A, we collect data on a single authoritative name server. The median number of name servers configured per DNS zone on that name server, however, is 4.0, which means that not all queries from Google for domains hosted on that name server will be sent to that particular name server. Typical resolver implementations will distribute queries over all authoritative name servers for a domain, usually favoring servers with shorter RTTs [14].

While it is infeasible to exhaustively determine RTTs from all Google PoPs to all authoritative name servers for domains for which our test server is also authoritative, we did want to get an idea how GPDNS resolvers factor in RTT when selecting an authoritative name server. Therefore, we conducted an experiment in which we setup four authoritative name servers for a single domain, each with a different public IPv4 address, but hosted on a single machine. This ensures uniform performance characteristics from an external viewpoint. We then measured the distribution of queries by GPDNS over several hours, where we artificially increase the RTT for one of the name servers every hour.

Figure 4 shows that a server which has an increased latency compared to the others, receives fewer queries. The ratio appears to be constant given a certain RTT distribution. In other words, as long as the latency remains constant, so does the distribution of queries over the authoritative name servers.

Based on this experiment, it is clear that by counting queries to our single vantage point, we cannot make claims about the total number of queries from GPDNS for domains for which our vantage point is authoritative. Since, however, the

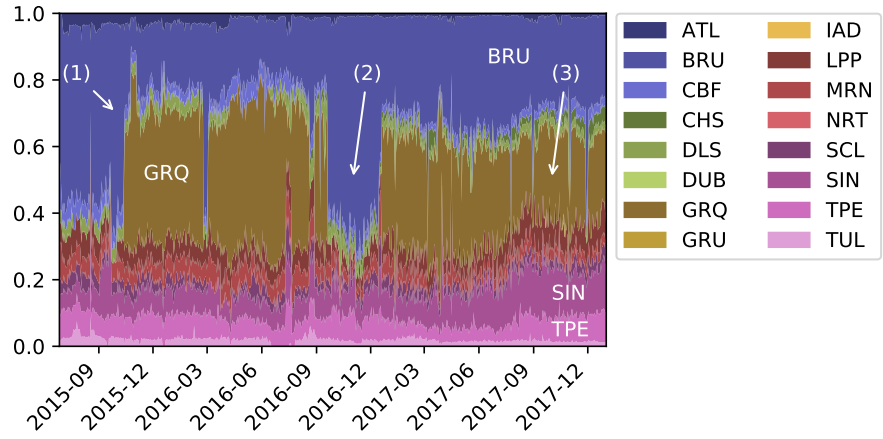

Figure 5. Ratio of DNS queries per PoP resolved via Google Public DNS

distribution of queries appears directly linked to RTT, and given that our vantage point is well-connected (a single hop away from major IXPs, including AMS-IX and LINX), we can measure trends in traffic coming from GPDNS over time.

\section{RESULTS}

\section{A. Query distribution}

The front-end IP address of GPDNS is 8.8 .8 .8 , however, as the service is anycasted, this means that an end-user can potentially reach any of the PoPs, as determined by BGP routing [15]. In this section, we look at the actual distribution of queries over the PoPs that are available. Figure 5 shows the relative distribution of the traffic over PoPs. The three letter acronyms in the legend indicate IATA airport codes. Since the authoritative DNS server where we captured the traffic is located in the Netherlands, and is authoritative for mostly Dutch domain names, we expect the PoPs near or in the Netherlands to handle most of the load.

Prior to October 2015 most traffic was handled in the BRU PoP (Brussels, Belgium). Then, when GRQ (Groningen, The Netherlands) was brought online, marked by (1) in the plot, there was a major shift. The traffic to the BRU PoP was significantly reduced at the same time, with all the other PoPs showing a reasonably constant amount of traffic. This is likely due to the fact that the majority of users in the Netherlands have a shorter path to GRQ than to BRU. In the period marked by (2), the situation temporarily reverted to its previous state as, for an unknown reason, the GRQ PoP was deactivated. We see that, as with the previous change, the amount of traffic handled by BRU PoP increases significantly.

After the GRQ PoP was re-enabled, in the period marked by (3), the distribution of traffic is largely stable with no significant changes in almost a year, other than a slow increase in the share of traffic from PoPs in Asia (TPE and SIN).

Figure 6 shows the distribution of the top-10 query types. The fraction of PTR records is surprisingly high, almost equivalent to the fraction of A queries. This can be explained by the fact that the authoritative name server on which we collected queries, is also responsible for over 2,100 reverse DNS zones. We suspect that most of these PTR queries are sent by mail servers, and examine this in more detail in Section IV-D. 
Table II

QUERIES ANSWERED OUT OF COUNTRY, WHILE AN IN-COUNTRY RESOLVER EXISTS. R = RESOLVER COUNTRY, C $=$ CLIENT COUNTRY

\begin{tabular}{|c|c|c|c|c|c|c|c|c|c|c|c|c|c|c|c|}
\hline \multirow[b]{2}{*}{ \# } & \multicolumn{3}{|c|}{$\begin{array}{c}\mathbf{A} \text { - before (1) } \\
1 \text { month before 2016-04-29 }\end{array}$} & \multicolumn{3}{|c|}{$\begin{array}{c}\text { B }- \text { during (1) } \\
1 \text { month after 2016-04-29 }\end{array}$} & \multicolumn{3}{|c|}{$\begin{array}{c}\mathbf{C}-\text { after (1), before (2) } \\
1 \text { month after } 2016-09-07\end{array}$} & \multicolumn{3}{|c|}{$\begin{array}{l}\text { D - during (2) } \\
1 \text { month before 2017-07-10 }\end{array}$} & \multicolumn{3}{|c|}{$\begin{array}{c}\mathbf{E}-\text { after }(2) \\
1 \text { month after 2017-07-10 }\end{array}$} \\
\hline & $\mathrm{R}$ & $\mathrm{C}$ & Count & $\mathrm{R}$ & $\mathrm{C}$ & Count & $\mathrm{R}$ & $\mathrm{C}$ & Count & $\mathrm{R}$ & $\mathrm{C}$ & Count & $\mathrm{R}$ & $\mathrm{C}$ & Count \\
\hline 1 & US & NL & $55,258,986$ & $\mathrm{NL}$ & $\mathrm{BE}$ & 825,902 & $\mathrm{BE}$ & NL & $33,545,772$ & $\mathrm{BE}$ & NL & $8,246,080$ & $\mathrm{BE}$ & GB & $18,155,553$ \\
\hline 2 & US & SG & $1,633,927$ & $\mathrm{BE}$ & IE & 733,032 & US & NL & $14,007,550$ & US & SG & $2,179,035$ & $\mathrm{BE}$ & $\mathrm{DE}$ & $4,011,011$ \\
\hline 3 & US & $\mathrm{BE}$ & 895,542 & $\mathrm{BE}$ & NL & 394,837 & JP & US & 966,666 & $\mathrm{BE}$ & IE & $1,035,833$ & US & BR & $3,092,432$ \\
\hline 4 & US & $\mathrm{IE}$ & 709,332 & TW & SG & 184,409 & $\mathrm{BE}$ & $\mathrm{IE}$ & 922,468 & $\mathrm{BE}$ & GB & 613,909 & SG & IN & $2,573,892$ \\
\hline 5 & US & TW & 520,368 & TW & US & 173,502 & TW & JP & 822,037 & TW & $\mathrm{JP}$ & 476,647 & $\mathrm{BE}$ & NL & $2,509,740$ \\
\hline
\end{tabular}

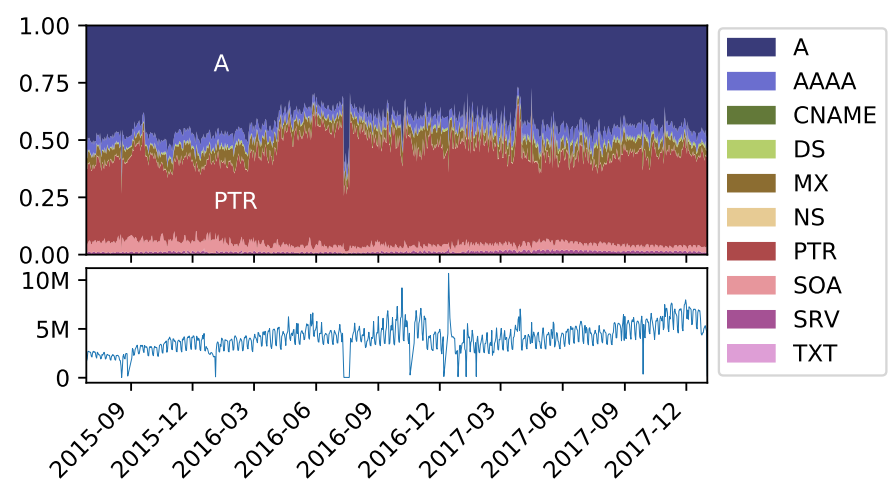

Figure 6. Ratio of DNS query types and absolute number of queries per day.

Lastly, we see no significant increase in AAAA queries (IPv6) over the full measurement period, indicating a surprising lack of uptake of IPv6 among clients of GPDNS.

\section{B. Out-of-country answers}

Earlier work [16] showed that for public DNS services such as GPDNS, the distance between a resolver and a client varies greatly. This can lead to performance penalties if the anycast PoP serving the client is geographically remote from the client. Another question to ask in this context is: are clients in a certain country always served by a PoP in that country? This is especially relevant in an age of ubiquitous surveillance by intelligence services, because if traffic is routed through or to another country, this exposes that traffic to potential prying eyes. With an anycasted service such as GPDNS, one might expect that if there is a PoP available in country $\mathrm{X}$, while making a request from that same country, that queries are answered from that PoP. However, depending on various parameters influencing BGP routing this is not necessarily the case.

Figure 7 shows the fraction of queries answered from outside the country of the end user, while a resolver inside was available. This is generally the case for $30 \%$ of queries. We observe two deviations from the overall trend, marked by (1) and (2), from late April 2016 to early September 2016 and from December 2016 to July 2017 respectively.

In Table II we show the top 5 queries that are answered out of country, while an in-country resolver was available. Queries are grouped by resolver country and client country. We compiled this top 5 over five time periods, A through E, each representing a month of data, either before or after the

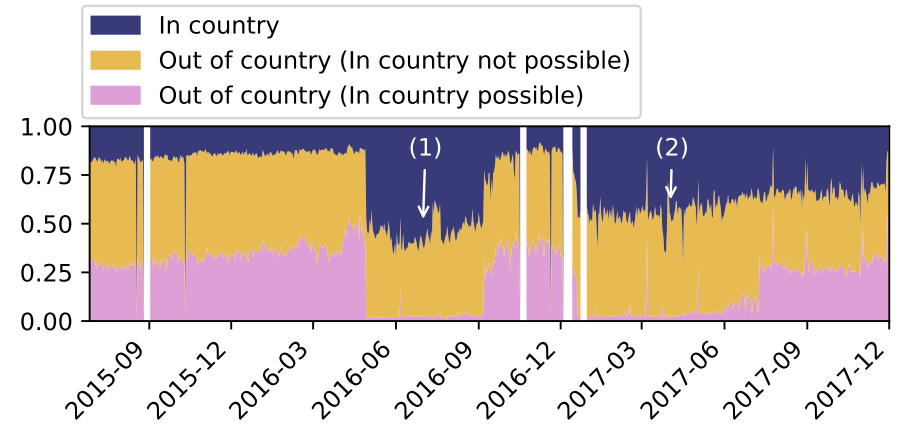

Figure 7. Ratio of out of country answers

beginning or end of one of the deviation periods (1) and (2), as labeled at the top of the table.

In period $\mathrm{A}$, before the start of event (1), the top 5 are all answered from the US, while at the same time the Brussels datacenter is clearly active (see Figure 5). Then, in period $\mathrm{B}$, during event (1), the total number of OOC queries drops dramatically, and the ones that do still occur are significantly closer in terms of geographic distance. The relative amount of OOC queries returns to its previous level in period $\mathrm{C}$, between events (1) and (2), although the distribution has changed significantly, arguably for the better (i.e., less geographical distance between resolver and client).

The changes that occur at the event marked as 2 are less dramatic, the number of clients who receive answers from a resolver in Belgium while located in Great Britain does increase significantly. The fact that these countries are relatively close to each other means that the performance impact is limited, although there is still a privacy impact. The situation for clients located in the Netherlands improved, as the number of queries served from Belgium decreased.

\section{Events leading to Google DNS adoption}

There are various reasons why an end-user might switch from their ISP's DNS resolvers to GPDNS, such as performance, security (in the form of DNSSEC) or resilience. In this section we take a closer look at an event that lead to a drastic increase in the use of GPDNS for a particular ISP.

The example we analyze in this section involves Ziggo, one of the largest ISPs in the Netherlands. Around August 2015 the DNS servers of this ISP suffered a Distributed Denialof-Service (DDoS) attack, causing serious service disruption for its customers. Major national news services (e.g., [17]) 


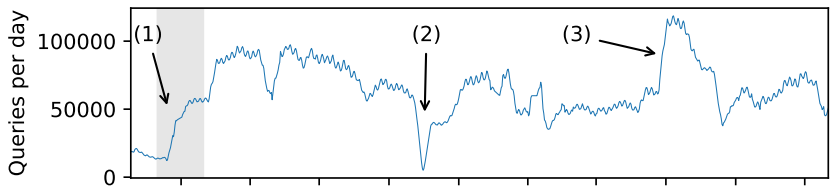

(a) Queries, moving average over -5 and +5 days

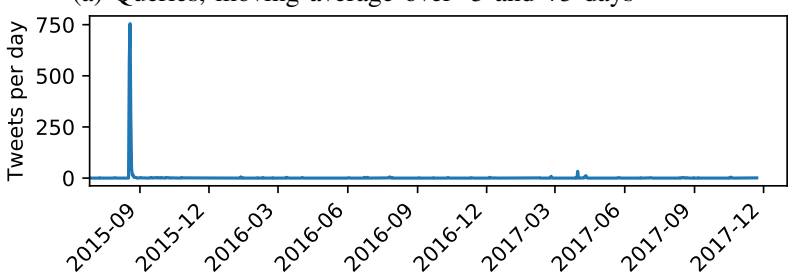

(b) Tweets mentioning DNS and Ziggo

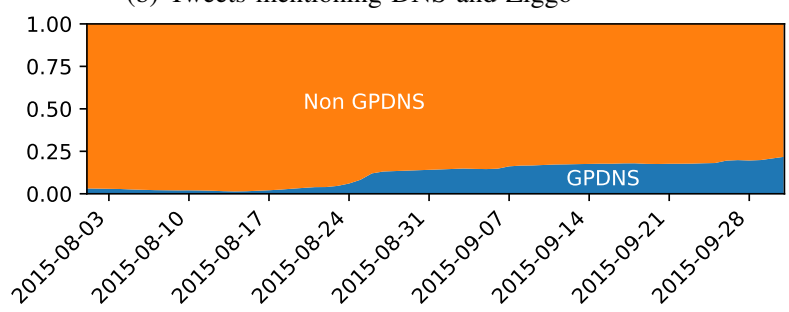

(c) Total queries (including Non-Google) from Ziggo

Figure 8. Ziggo (AS9143)

reported that configuring a third-party DNS service could help users. We asked ourselves: does an attack on a major provider and subsequent media coverage suggesting the use of e.g., GPDNS lead to increased adoption of GPDNS?

As it turns out, this is indeed the case. In Figure 8a we show the number of queries that originate from Ziggo's AS (9143) per day, using a moving average of -5 to +5 days. The uptake around the date of the attack can be clearly seen, marked by (1). The event indicated by (2) indicates a gap in our data collection, while (3) is caused by a single / 24 temporarily issuing tens of thousands of requests. Figure $8 \mathrm{~b}$ shows the number of tweets that used the words DNS and Ziggo over the entire measurement period. The tweets were collected using Twitter's web API. The clear spike coincides with the DDoS attack, and marks the beginning of the increase in GPDNS use.

Another takeaway from Figure 8a is that uptake remains high, even after the attack has passed and Ziggo's DNS servers return to normal operation. This shows that, while DNS is a fairly technical concept, in case of a major outage such as this, people will switch away from their ISP's DNS servers, and more importantly: never switch back. How dramatic this effect really is, is illustrated by Figure 8c. This graph (which zooms in on the two-month period around the attack) shows what fraction of queries to our vantage point arrive directly from Ziggo's AS, and what fraction arrives through Google.

\section{SMTP, Google, and EDNSO Client Subnet}

As we hinted at in Section IV-A, the distribution of query types shows a large percentage of PTR queries. Pointer (PTR) records are used to define reverse DNS names for IP addresses. For example, given IP address 10.0.0.1 there might be a PTR entry for 1.0.0.10.in-addr.arpa which points to a hostname, for example my.host.com.

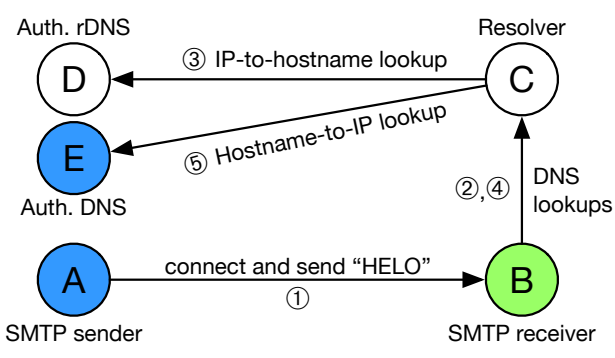

Figure 9. SMTP DNS Lookup process

For a complete configuration there should then also be an A record for my.host.com that resolves to 10.0.0.1. Using this methodology an IP address can be converted to its corresponding hostname and vice versa.

Reverse DNS (rDNS) is commonly used by mail servers to authenticate a sending host. Upon an incoming connection, an SMTP server typically performs a lookup of the reverse hostname of the connecting IP. If a hostname is returned, it will subsequently attempt to resolve this hostname back to the corresponding IP address. In standard configurations SMTP servers may not accept e-mail from other SMTP servers if they do not have a correctly configured reverse hostname [18]. We have verified that at least Postfix has implemented this functionality.

The fact that we see large numbers of PTR queries coming via Google, suggests that there may be SMTP servers that have configured GPDNS as their resolver. This is a potential privacy issue, as an SMTP server then discloses to Google which servers are connecting to it to deliver e-mail. Even worse, though, GPDNS may in turn disclose this information to authoritative name servers through the ECS extension. While intuitively one might think this is not a serious issue, consider that these queries are not only sent to the authoritative name server for a domain or reverse DNS zone, but also to the name servers of their parent domain. Concretely, this exposes information about e-mail traffic to TLD and Root operators, as well as reverse DNS operators higher up the DNS hierarchy.

Figure 9 illustrates this scenario. In step (1) a sending SMTP server A connects to a receiving SMTP server B. In step (2), $\mathrm{B}$ then performs a reverse DNS lookup for the IP address of A via resolver C (which could be Google Public DNS). Resolver $\mathrm{C}$ resolves the actual IP address to a hostname by contacting the authoritative nameserver D in step (3). Once the reverse hostname is known usually the reverse hostname is again resolved to an IP address, following a similar pattern as before, in steps (4) and (5). If GPDNS is used, it may include ECS information in the queries to name servers $\mathrm{D}$ and $\mathrm{E}$ (if they support ECS), exposing information about which host is sending mail to this mail server to the authoritative name server operators. This becomes worse if GPDNS does not have sufficient information in its cache to contact D and E directly, and first needs to perform a full DNS recursion, hitting servers further up the DNS hierarchy (TLD, root, ... ). If these also support ECS, information also leaks to these parties. Table III describes for different scenarios what information is leaked. 
Table III

INFORMATION LEAK SCHEMA

\begin{tabular}{lll}
\hline & \multicolumn{2}{c}{ Google DNS at Sender } \\
\cline { 2 - 3 } Operator of service below can see & About sender & About receiver \\
\hline Google DNS* & IP (Query source) & IP (DNS record) \\
Authoritative DNS for Reverse** & $\mathrm{n} / \mathrm{a}$ & $\mathrm{n} / \mathrm{a}$ \\
Intermediate DNS** & $\mathrm{n} / \mathrm{a}$ & $\mathrm{n} / \mathrm{a}$ \\
Authoritative DNS for Forward** & $/ 24$ prefix (ECS) & IP (DNS record) \\
Intermediate DNS** & /24 prefix (ECS) & IP (DNS record) \\
\hline & \multicolumn{1}{c}{ Google DNS at Receiver } \\
\cline { 2 - 3 } Operator of service below can see & About sender & About receiver \\
\hline Google DNS* & IP (DNS record) & IP (Query source) \\
Authoritative DNS for Reverse** & IP (DNS record) & /24 prefix (ECS) \\
Intermediate DNS** & IP (DNS record) & /24 prefix (ECS) \\
Authoritative DNS for Forward** & IP (DNS record) & $/ 24$ prefix (ECS) \\
Intermediate DNS** & IP (DNS record) & /24 prefix (ECS) \\
\hline$*$ Unless already in local cache (subject to TTL), & \\
$* *$ Unless already cached by Google (Subject to TTL) & \\
\hline
\end{tabular}

In order to verify this scenario in practice, we first extracted the subnets responsible for the bulk (80\%) of the PTR queries, resulting in approximately 2,000/24 subnets. We then used a standard scanning tool (nmap) to find systems which had port 25 (most likely SMTP) open. The scan found that a little over $50 \%$ of the subnets contain at least one system listening on port 25 , for a total of roughly $15 \mathrm{k}$ systems.

We connect to each of the systems that have port 25 open, with a timeout of 3 seconds. We immediately transmit our identity in the client initiation phase of the SMTP session. We then read data for 6 seconds, checking for SMTP status codes as specified in [19], or until we receive a 250 message, indicating that our HELO message has been accepted. To determine if the SMTP daemon uses Google Public DNS to lookup the reverse hostname of our connecting system, we monitor the incoming DNS queries on system E in Figure 9. If we see an incoming DNS query for our domain between the time of connecting and the time of disconnecting we assume that this DNS query is a result of our connection. While we cannot be certain that it is in fact the SMTP daemon itself that performs the lookup as opposed to, for example, the firewall, this makes no difference to the privacy risks.

Table IV summarizes our results. Of the approximately 10k SMTP servers that we found that transmitted a valid status (a 3 digit number at the beginning of a line), we saw an incoming DNS query from roughly two thirds, and half of those came through GPDNS. We repeated the experiment without sending an initial HELO message from our side, with similar results.

For comparison, we also scanned the top 2,000/24s responsible for MX queries. In contrast to PTR queries, these are likely to originate from "sending" SMTP servers. We find approximately $43 \mathrm{k}$ systems with this scan. Similar to our previous results, approximately two thirds of these perform a DNS query on connection, and half do this via GPDNS.

Summarizing, SMTP servers that (indirectly) use GPDNS as a resolver are common. Worryingly, we find 14,204 SMTP servers that, upon connection, leak our IP address or our prefix to GPDNS and any DNS servers that are hit during recursion.
Table IV

RESULTS OF CONNECTING TO EACH OF THE SMTP SERVERS

\begin{tabular}{|c|c|c|}
\hline & IPs (PTR) & IPs (MX) \\
\hline Connectable & 15.374 & 42.693 \\
\hline Valid SMTP Status code & 9.681 & 32.391 \\
\hline DNS query in timeframe & 6.503 & 20.107 \\
\hline From Google AS & 3.188 & 11.208 \\
\hline Total unique & \multicolumn{2}{|c|}{14.204} \\
\hline Total DNS queries received & 11.076 & 27.723 \\
\hline
\end{tabular}

\section{RELATED WORK}

The idea for the EDNS Client Subnet extension was first tabled in 2011, supported by a coalition of parties promoting a "Faster Internet" . Partners in this project include both CDN operators and operators of large public DNS resolvers.

Otto et al. were the first to study ECS. In their paper [3], they study the impact of the use of public DNS resolvers on web $\mathrm{CDN}$ performance, and highlight the performance improvement ECS could offer in this context. Furthermore, they study the first preliminary uptake of ECS by CDN operators. In follow-up work [4], Sánchez et al. study the performance improvement of CDN web delivery if ECS is used via Google Public DNS. Streibelt et al. use ECS to study the infrastructure of CDNs that support ECS. Their paper [5] shows how ECS can be used to provide insight into CDN server deployments, and CDN serverto-client mappings. They highlight that they can perform such mappings from a single vantage point, by inserting arbitrary prefixes in the scope field of an ECS query, provided that CDN operators do not limit from which sources they are willing to respond to ECS-enabled DNS queries. Additionally, Streibelt et al. also study aggregation by ECS-enabled CDNs, showing different strategies where some CDNs return ECS responses with larger scopes (i.e. returning an ECS response for an IPv4 /16 prefix when a smaller / 24 prefix is specified in the DNS query), whereas others respond with narrower scopes than asked for, going as low as a /32. The authors speculate that CDNs that follow the latter practice essentially want to force DNS resolvers to cache the result only for a single client.

Calder et al. use ECS for a longitudinal study of Google's service delivery CDN. Their work [6] shows that using ECS they can create a complete mapping of this $\mathrm{CDN}$ and can uncover dramatic growth of this CDN over a ten-month period. Fan et al. also use ECS to study Google's CDN, but rather than focusing on the CDN infrastructure itself, they study changes in client prefix to CDN front-end mappings over time [7].

Chen et al. study the impact of ECS deployment from inside the Akamai CDN [16]. The introduction of ECS at Akamai resulted in a 30\% improvement in startup time for connections to the CDN, at the cost of an eight-fold increase in the number of DNS queries to their name servers. Chen et al. are the first to show the RTT performance penalty incurred by users due to their DNS requests getting routed to geographically remote public resolvers. In this work we significantly extend on this

\footnotetext{
${ }^{3}$ http://www.afasterinternet.com/participants.htm
} 
by using longitudinal data covering 2.5 years, showing, e.g., changes in out-of-country query handling over time.

A common denominator of the related work to date has been that it exclusively focused on using ECS to study service delivery by CDNs or to study how ECS can improve this service delivery. In contrast, in this work, we leverage ECS to study the behavior of and use of a large public DNS provider.

Finally, Kintis et al. discuss some of the privacy implications of ECS [20]. Their focus is the privacy risks imposed by on-path attackers between the public DNS resolver and authoritative name servers on the Internet. They observe how an on-path attacker can perform selective surveillance on clients of public DNS resolvers. In addition to this, they also show how an attacker can selectively poison a public DNS resolver's cache for specific clients using ECS. In this work, we extend this by showing new privacy risks where the use of public DNS resolvers by SMTP servers to perform DNS resolution leaks information about the IP addresses and domains of hosts sending e-mail to these servers.

\section{CONCLUSIONS}

There has been much debate about the privacy risks of using public DNS resolvers. The obvious argument is that the operator of such a resolver gets access to extremely privacy-sensitive information in the form of DNS queries. One aspect of privacy in the context of public DNS resolvers remains underexposed. In order for CDNs to be able to make Geo IP-based decisions, many public resolvers use a DNS extension called EDNS0 Client Subnet (ECS), which allows them to reveal part of a client's IP to the CDN. In essence, the need for ECS is an unintended side-effect of the use of public DNS resolvers.

Earlier work leveraged ECS to study content delivery networks. In this paper we show that ECS can also be used to study the day-to-day operations of a public DNS resolver, in our case GPDNS. This allowed us to show that traffic to GPDNS is frequently routed to out-of-country GPDNS PoPs for weeks at a time, even though an in-country PoP is available. This potentially exposes DNS traffic to state-level surveillance. We also showed that certain events such as DDoS attacks on ISP DNS resolvers cause users to switch to GPDNS en masse, and, more importantly, once users have switched to Google they do not switch back.

A previously unrecognized privacy issue is that e-mail servers frequently use GPDNS for DNS resolution. Obviously, this reveals information to Google where these servers receive mail from and send mail to. Much more insidious though, is, that this information also leaks to operators of authoritative name servers through Google's use of ECS. Where previously mail servers were hidden behind DNS resolvers of network operators, they are now exposed up to the / 24 IP prefix level for IPv4, and / 56 for IPv6 (was / 64 until the 13th of December 2017).

Taken together, we can conclude that not only should the use of public DNS resolvers in general be questioned; given the privacy implications, the use of the ECS DNS extension introduced specifically for public DNS resolvers should also be re-examined. Given our findings, we strongly advocate restricting use of ECS toward content delivery networks only, on an opt-in basis, to prevent leaking unnecessary information.

\section{ACKNOWLEDGMENTS}

Work at the University of Twente was supported by SURFnet Research on Networks. This paper used IP2Location LITE data available from https://lite.ip2location.com.

\section{REFERENCES}

[1] Google, "Google Public DNS and Location-Sensitive DNS Responses," 2014. [Online]. Available: https://webmasters.googleblog.com/2014/12/ google-public-dns-and-location.html

[2] C. Contavalli, W. van der Gaast, D. Lawrence, and W. Kumari, "RFC 7871 - Client Subnet in DNS Queries," 2016. [Online]. Available: https://tools.ietf.org/html/rfc7871

[3] J. S. Otto, M. A. Sánchez, J. P. Rula, and F. E. Bustamante, "Content Delivery and the Natural Evolution of DNS: Remote DNS Trends, Performance Issues and Alternative Solutions," in Proceedings of ACM IMC 2012. Boston, MA, USA: ACM Press, 2012, pp. 523-536.

[4] M. Sánchez, J. S. Otto, Z. S. Bischof, D. Choffnes, F. Bustamante, B. Krishnamurthy, and W. Willinger, "Dasu: Pushing Experiments to the Internet's Edge," in Proceedings of USENIX NSDI 2013. Lombard, IL, USA: USENIX Association, 2013, pp. 487-499.

[5] F. Streibelt, J. Böttger, N. Chatzis, G. Smaragdakis, and A. Feldmann, "Exploring EDNS-Client-Subnet Adopters in Your Free Time," in Proceedings of ACM IMC 2013. Barcelona, Spain: ACM Press, 2013, pp. 305-312.

[6] M. Calder, X. Fan, Z. Hu, E. Katz-Bassett, J. Heidemann, and R. Govindan, "Mapping the Expansion of Google's Serving Infrastructure," in Proceedings of ACM IMC 2013. Barcelona, Spain: ACM Press, 2013, pp. 313-326.

[7] X. Fan, E. Katz-Bassett, and J. Heidemann, "Assessing Affinity Between Users and CDN Sites," in Traffic Measurements and Analysis, ser. LNCS, M. Steiner, P. Barlet-Ros, and O. Bonaventure, Eds. Barcelona, Spain: Springer Berlin Heidelberg, 2015, vol. 9053, pp. 95-110.

[8] D. Giordano, D. Cicalese, A. Finamore, M. Mellia, M. Munafo, D. Joumblatt, and D. Rossi, "A First Characterization of Anycast Traffic from Passive Traces," in IFIP workshop on Traffic Monitoring and Analysis (TMA), 2016, pp. 30-38.

[9] P. Mockapetris, "RFC 1034 - Domain Names - Concepts and Facilities," 1987. [Online]. Available: https://tools.ietf.org/html/rfc1034

[10] — "RFC 1035 - Domain Names - Implementation and Specification," 1987. [Online]. Available: http://tools.ietf.org/html//rfc1035

[11] J. Damas, M. Graff, and P. Vixie, "RFC 6891 - Extension Mechanisms for DNS (EDNS0)," 2013. [Online]. Available: http: //tools.ietf.org/html/rfc6891

[12] CAIDA, "The CAIDA UCSD Routeviews Prefix to AS mappings Dataset (pfx2as) for IPv4 and IPv6," 2018. [Online]. Available: http://www.caida.org/data/routing/routeviews-prefix2as.xml

[13] Google, "Google Public DNS FAQ," 2018. [Online]. Available: https://developers.google.com/speed/public-dns/faq\#locations

[14] Y. Yu, D. Wessels, M. Larson, and L. Zhang, "Authority Server Selection in DNS Caching Resolvers," ACM SIGCOMM Computer Communication Review, vol. 42, no. 2, p. 80, 2012.

[15] W. B. de Vries, R. de O Schmidt, W. Hardaker, J. Heidemann, P.-T de Boer, and A. Pras, "Broad and Load-Aware Anycast Mapping with Verfploeter," in Proceedings of ACM IMC 2017. ACM, 2017, pp. 477-488.

[16] F. Chen, R. K. Sitaraman, and M. Torres, "End-User Mapping: Next Generation Request Routing for Content Delivery," in Proceedings of ACM SIGCOMM 2015. London, UK: ACM Press, 2015, pp. 167-181.

[17] NOS, "Is your provider under attack? This is how you restore service [in Dutch]," Aug 2015. [Online]. Available: https://nos.nl/op3/artikel/ 2052944-aanval-op-je-provider-zo-krijg-je-weer-verbinding.html

[18] M. Kucherawy, "RFC 7601 - Message Header Field for Indicating Message Authentication Status," 2015. [Online]. Available: https: //tools.ietf.org/html/rfc7601

[19] J. Klensin, "RFC 5321 - Simple Mail Transfer Protocol," 2008. [Online]. Available: https://tools.ietf.org/html/rfc5321

[20] P. Kintis, Y. Nadji, D. Dagon, M. Farrell, and M. Antonakakis, "Understanding the Privacy Implications of ECS," in Proceedings of DIMVA 2016, ser. LNCS, J. Caballero, U. Zurutuza, and R. Rodríguez, Eds. Springer Berlin Heidelberg, 2016, vol. 9721, pp. 343-353. 